\section{Chemotherapy-induced antitumor immunity requires formyl peptide receptor 1}

\author{
Erika Vacchelli, ${ }^{1,2,3,4,5 *}$ Yuting $M a,{ }^{1,2,3,4,5,6,7 *}$ Elisa E. Baracco, ${ }^{1,2,3,8}$ \\ Antonella Sistigu, ${ }^{9}$ David P. Enot, ${ }^{1,2,3,10}$ Federico Pietrocola, ${ }^{1,2,3,8}$ Heng \\ Yang, ${ }^{1,2,3,4,5,6,7}$ Sandy Adjemian, ${ }^{1,2,3}$ Kariman Chaba, ${ }^{1,2,3,4}$ Michaela \\ Semeraro, ${ }^{1,11,12}$ Michele Signore, ${ }^{13}$ Adele De Ninno, ${ }^{14}$ Valeria Lucarini, ${ }^{13}$ \\ Francesca Peschiaroli, ${ }^{13}$ Luca Businaro, ${ }^{14}$ Annamaria Gerardino, ${ }^{14}$ \\ Gwenola Manic, ${ }^{9}$ Thomas Ulas, ${ }^{15}$ Patrick Günther, ${ }^{15}$ Joachim L. \\ Schultze, ${ }^{15}$ Oliver Kepp, ${ }^{1,2,3,4,5}$ Gautier Stoll, ,2,3,4,5 Céline Lefebvre, ${ }^{1,16}$ \\ Claire Mulot, ${ }^{17,18}$ Francesca Castoldi, ${ }^{1,2,3,8,19}$ Sylvie Rusakiewicz, ${ }^{1,11,12}$ \\ Sylvain Ladoire, ${ }^{20,21,22}$ Lionel Apetoh, ${ }^{20,21,22}$ José Manuel Bravo-San \\ Pedro, ${ }^{1,2,3,4,5}$ Monica Lucattelli, ${ }^{23}$ Cécile Delarasse,${ }^{24}$ Valérie Boige,${ }^{18,25}$ \\ Michel Ducreux, ${ }^{8,25}$ Suzette Delaloge, ${ }^{16,26}$ Christophe Borg, ${ }^{27}$ Fabrice \\ André,,16,28,29 Giovanna Schiavoni, ${ }^{13}$ Ilo Vitale, ${ }^{9,30}$ Pierre Laurent- \\ Puig, ${ }^{17,18,31}$ Fabrizio Mattei, ${ }^{13 \dagger}$ Laurence Zitvogel, ${ }^{1,8,11,12+{ }^{+}}$Guido \\ Kroemer ${ }^{1,2,3,4,5,10,31,32+*}$
}

${ }^{1}$ Gustave Roussy Cancer Campus, Villejuif, France. ${ }^{2}$ INSERM, U1138, Paris, France. ${ }^{3}$ Equipe 11 labellisée par la Ligue Nationale contre le Cancer, Centre de Recherche des Cordeliers, Paris, France. ${ }^{4}$ Université Paris Descartes, Sorbonne Paris Cité, Paris, France. ${ }^{5}$ Université Pierre et Marie Curie, Paris, France. ${ }^{6}$ Suzhou Institute of Systems Medicine, Suzhou, Jiangsu, China. ${ }^{7}$ Chinese Academy of Medical Sciences and Peking Union Medical College, Beijing, China. ${ }^{8}$ Faculté de Médecine, Université Paris-Saclay, Kremlin-Bicêtre, France. ${ }^{9}$ Regina Elena National Cancer Institute, Rome, Italy. ${ }^{10}$ Metabolomics and Cell Biology Platforms, Gustave Roussy Cancer Campus, Villejuif, France. ${ }^{11}$ INSERM, U1015, Villejuif, France. ${ }^{12}$ Center of Clinical Investigations in Biotherapies of Cancer (CICBT) 1428, Villejuif, France. ${ }^{13}$ Department of Hematology, Oncology and Molecular Medicine, Istituto Superiore di Sanità, Rome, Italy. ${ }^{14}$ Italian National Research Council, Institute for Photonics and Nanotechnology, Rome, Italy. ${ }^{15}$ Genomics and Immunoregulation, Life and Medical Science Center Institute, University of Bonn, Germany. ${ }^{16}$ INSERM, U981, Villejuif, France. ${ }^{17}$ Université Paris Sorbonne Cité, UMRS 775, INSERM, Paris, France. ${ }^{18}$ INSERM U1147, Centre de ressources biologiques (CRB) EPIGENETIC, Paris, France. ${ }^{19}$ Sotio a.c., Prague, Czech Republic. ${ }^{20}$ Department of Medical Oncology, Centre Georges-François Leclerc, Dijon, France. ${ }^{21}$ Université Bourgogne Franche-Comté, Dijon, France. ${ }^{22}$ Centre Georges François Leclerc, Dijon, France. ${ }^{23}$ Department of Life Sciences, University of Siena, Siena, Italy. ${ }^{24}$ Institut du Cerveau et de la Moelle épinière, ICM CNRS UMR 7225 - INSERM U 1127 - UPMC-P6 UMR S 1127, Équipe Neurogénétique et Physiologie Hôpital de la Pitié-Salpêtrière, 47. Boulevard de l'Hôpital, 75013 Paris, France. ${ }^{25}$ Department of Medical Oncology, Gustave Roussy Cancer Campus, Villejuif Cedex, France. ${ }^{26}$ Department of Breast Oncology, Gustave Roussy Cancer Campus, Villejuif, France. ${ }^{27}$ University of Franche-Comté, INSERM 1098, France. ${ }^{28}$ Department of Biology and Pathology, Gustave Roussy Cancer Campus, Villejuif, France. ${ }^{29}$ Department of Medical Oncology, Gustave Roussy Cancer Campus, Villejuif, France. ${ }^{30}$ Department of Biology, University of Rome “Tor Vergata," Rome, Italy. ${ }^{31}$ Pôle de Biologie, Hôpital Européen Georges Pompidou, AP-HP, Paris, France. ${ }^{32}$ Karolinska Institute, Department of Women's and Children's Health, Karolinska University Hospital, 17176 Stockholm, Sweden.

*These authors contributed equally to this work.

${ }^{\dagger}$ These authors contributed equally to this work.

†Corresponding author. E-mail: kroemer@orange.fr (G.K.); laurence.zitvogel@gustaveroussy.fr (L.Z.)

Abstract. Antitumor immunity driven by intratumoral dendritic cells contributes to the efficacy of anthracycline-based chemotherapy in cancer. We identified a lossof-function allele of the gene coding for formyl peptide receptor 1 (FPR1) that was associated with poor metastasis-free and overall survival in breast and colorectal cancer patients receiving adjuvant chemotherapy. The therapeutic effects of anthracyclines were abrogated in tumor-bearing $F p r 1^{-/-}$mice due to impaired antitumor immunity. Fpr1-deficient dendritic cells failed to approach dying cancer cells and as a result could not elicit antitumor $T$ cell immunity. Experiments performed in a microfluidic device confirmed that FPR1 and its ligand, annexin-1, promoted stable interactions between dying cancer cells and human or murine leukocytes. Altogether, these results highlight the importance of FPR1 in chemotherapy-induced anticancer immune responses.

The success of anticancer chemotherapy is linked to a durable tumor-targeting immune response (1). Accordingly, the presence of tumor-infiltrating dendritic cells (DCs) and $\mathrm{CD}^{+} \mathrm{T}$ lymphocytes at diagnosis increases the likelihood of breast cancer patients to respond to anthracyclines (2-5) (6). One mechanism through which anthracyclines can stimulate an antitumor immunity is by inducing immunogenic cell death (ICD), and this mechanism implies signaling through innate immune receptors including tolllike receptors 3 (TLR3) and 4 (TLR4) (7-10).

We designed a screen for identifying candidate genetic defects that negatively affect chemotherapeutic responses. To this aim, we identified 328 nonsynonymous single nucleotide polymorphisms (SNPs) affecting proteins involved in the recognition of dying cells by innate immune effectors (11) or influencing the incidence or prognosis of major human cancers, and occurring in Caucasians with an allelic frequency $>5 \%$ (table S1). DNA samples from breast cancer patients treated with adjuvant anthracycline-based chemotherapy (belonging to two independent cohorts, table S2 and S3) were genotyped, and the effect of each SNP on overall survival (OS) was determined. When a dominant genetic model was applied, only one SNP was significantly (raw $P$ $<0.05$ ) associated with OS independent of major clinicopathological variables in both patient cohorts: rs867228 (fig. S1A, C to $H)$. This SNP $(1037 \mathrm{~A}>\mathrm{C})$ affects exon 2 of the gene coding for formyl peptide receptor 1 (FPRI), which promotes the chemotactic interaction of neutrophils with necrotic hepatocytes $(12,13)$. rs867228 provokes an amino acid substitution (E346A) that suppresses FPR1 signaling (14, 15). Other SNPs with less drastic effects on FPR1 function $(15,16)$ had no impact on OS, (fig. S1B and fig. S2, A to C). As opposed to patients bearing the most frequent FPR1 allele (17) in homozygosis $\left(F P R I^{C C}\right)$, women bearing the rs867228 lossof-function allele of $F P R 1$ in homozygosis $\left(F P R 1^{A A}\right)$ or heter- 
ozygosis $\left(F P R 1^{C A}\right)$ exhibited reduced OS and metastasis-free survival (Fig. 1, A and B; fig. S2, D and E; and tables S4 and S5). Similarly, colorectal cancer patients treated with oxaliplatin-based chemotherapy (table S6) and bearing the rs867228 loss-of-function allele of FPR1 in homozygosis $\left(F P R 1^{A A}\right)$, exhibited reduced OS and progression-free survival (fig. S3, A to D, and tables S7 and S8). rs867228 had no impact on the OS of breast cancer patients bearing loss-offunction alleles of TLR4 (rs4986790, D299G) (7, 18) (Fig. 1C and table S9) or TLR3 (rs3775291, L412F) (Fig. 1E and table S10). The negative impact of the FPR1 polymorphism on OS was only evident in patients with normal TLR3 and TLR4 (Fig. 1, D and F, and tables S9 and S10), suggesting that FPR1 participates in the same therapeutically relevant pathway as that one influenced by the two TLRs.

FPR1 is expressed by myeloid cells (http://www.proteinatlas.org/; https://www.immgen.org/) as well as by some carcinomas (19). Genetic inactivation of Fpr1 (fig. S4A) did not affect the capacity of MCA205 fibrosarcoma cells treated with mitoxantrone or doxorubicin in vitro to vaccinate syngeneic, immunocompetent mice against a re-challenge with living cells of the same type (Fig. 2, A and B, and fig. S4A). Similar results were obtained with WT and $F_{p r 1^{-/-}}$TC-1 lung carcinoma cells (fig. S4A, fig. S6, A and B, and fig. S7, A and B). However, Fpr1 ${ }^{-/-}$mice failed to mount an immune response against anthracycline-treated MCA205 (Fig. 2, A and B, and fig. S5A) or TC-1 cells (fig. S6, $A$ and B, and fig. S7, A and B) under conditions in which WT mice did so, suggesting that Fpr1 must be expressed by host cells, not cancer cells, for anthracyclines to elicit antitumor immunity. Accordingly, Fpri ${ }^{-/-}$cancer cells responded to anthracycline-based chemotherapy in vivo as WT cancer cells did, whereas WT cancer cells growing in $\mathrm{FprI}^{-1-}$ hosts were resistant to anthracyclines (Fig. 2, C and D, and figs. S5B, S6C, S6D, S7C, and S7D).

Next, we investigated how FPR1 may influence the immune response against dying cancer cells by making bone marrow chimeras. WT mice receiving $\mathrm{FprI}^{-{ }^{-}}$bone marrow could not mount a protective immune response after vaccination with anthracycline-treated MCA205 cells (fig. S8A). Moreover, MCA205 cell-derived tumors became refractory to chemotherapy when they grew in WT mice that had received $\mathrm{FprI}^{-1-}$ bone marrow (fig. S8B). Conversely, tumors growing in $\mathrm{FprI}^{-/-}$hosts reconstituted with WT bone marrow responded normally to anthracycline-based chemotherapy (fig. S8C). Thus, expression of FPR1 by the host immune system contributes to the antineoplastic effects of chemotherapy.

Four ligands for FPR1 have been described, namely (i) cathepsin G (CTSG) (20), (ii) family with sequence similarity 19 [chemokine (C-C motif)-like], member A4 (FAM19A4) (21), (iii) $N$-formylated peptides contained in bacteria and mitochondria (22), and (iv) annexin A1 (ANXA1), an ubiquitously expressed cytosolic protein (23). Knocking out the genes coding for Ctsg (fig. S4D), Fam19a4 (fig. S4E) or mito- chondrial methionyl-tRNA formyltransferase (Mtfmt), an enzyme that catalyzes formylation reactions in mitochondria (24) (fig. S4B), had no effect on the capacity of MCA205 cells to elicit anticancer immune responses upon exposure to anthracyclines in vitro, and to respond to mitoxantrone and doxorubicin in vivo (Fig. 2, E and F, fig. S5, C and D, fig. S6, E to H, fig. S7, E to H, fig. S8, D and E, and fig. S9). Conversely, anthracycline-treated Anxa1 ${ }^{-/-}$MCA205 and TC-1 cells (fig. S4C) were unable to drive protective, tumortargeting immune responses and formed tumors that were resistant to doxorubicin and mitoxantrone (Fig. 2, E and F, fig. S5, C and D, fig. S6, E to H, fig. S7, E to H, fig. S8, D and E, and fig. S9). Both anthracyclines stimulated the secretion of Anxa1 by cancer cells (fig. S4F). Moreover, blockade of extracellular ANXA1 with a neutralizing antibody abrogated tumor growth reduction by anthracyclines in vivo (fig. S10), supporting the idea that ANXA1 is the functionally relevant FPR1 ligand.

Injection of the FPR1-specific antagonist cyclosporin $\mathrm{H}$ (CsH) (25) either concomitant with chemotherapy, or 2 (but not 7) days after chemotherapy, abolished the anticancer effects of anthracyclines (fig. S11). Thus, FPR1 is likely to participate in the initial phase of the anticancer immune response. Microarray analyses of tumors collected from WT versus FprI $^{-1-}$ mice 2 days post-chemotherapy led to the identification of multiple genes that were induced by doxorubicin in WT (but not in $\mathrm{FprI}^{-/-}$) hosts (Fig. 3, A to C, fig. S12, and table S11). These genes included several that are relevant for type I IFN responses, DC maturation and antigen processing/presentation, and cytotoxic T-cell effector functions. Fpr1 was expressed by intratumoral CD45 leukocytes (not by $\mathrm{CD}_{45}^{-}$cells), in particular myeloid Ly6 ${ }^{\text {high }} \mathrm{Ly} 6 \mathrm{G}^{-}$and $\mathrm{Ly} 6 \mathrm{G}^{+}$cells post-chemotherapy in vivo (Fig. 3B and fig. S13, A to D). Moreover, Fpr1 expression levels increased in bone marrow-derived DC confronted with dying (but not living) cancer cells in vitro (fig. S13, E to G). Ly6C ${ }^{\text {high }}{ }^{2 y} 6 \mathrm{G}^{-}$cells do (while $\mathrm{Ly} 6 \mathrm{G}^{+}$cells do not) contribute to the efficacy of anthracycline-based chemotherapy (26). Our results therefore suggest the importance of FPR1 expression in the Ly6 $\mathrm{C}^{\text {high }} \mathrm{Ly}_{6 \mathrm{G}^{-}}$myeloid cell population, which comprises DCs and their precursors (26). Elevated expression of the human orthologs of all immune-relevant genes influenced by Fpr1 in mice (Fig. 3A) had a significant $(P<$ 0.05) positive impact on the response of breast cancer patients to anthracyclines in 4 out of 5 cohorts studied (Fig. $3 \mathrm{D})$, suggesting that these genes are clinically relevant. Moreover, the mutational status of FPR1 influenced the expression level of several genes involved in antigen presentation within neoplastic lesions of breast cancer patients (fig. S14).

The presence or absence of Anxal (in tumor cells) or that of Fprl (in the host) did not impact on the capacity of chemotherapy to elicit autophagy, apoptosis and necrosis in cancer cells (fig. S15). Moreover, the absence of Fprl in the host did not influence the density of tumor infiltration by 


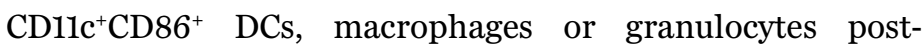
chemotherapy (fig. S16A). Morphometric analyses of the ratio between the maximum length and the perpendicular width of tumor-infiltrating myeloid cells, reflecting cellular asymmetry related to migration (27), revealed a selective defect in the chemotherapy-induced elongation of intratumoral DCs (but not macrophages and granulocytes) in $\mathrm{FprI}^{-1-}$ hosts (Fig. 3, E to G, and figs. S16B and S17). Two days after chemotherapy, a sizeable fraction of cancer cells underwent apoptosis and hence contained immunofluorescence microscopy-detectable active caspase 3 (Casp3a). In

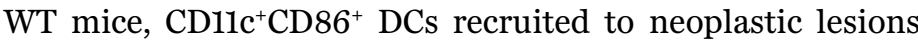
by chemotherapy were closer to the most proximal Casp3 $\mathrm{a}^{+}$ (dying) cancer cell than to the nearest Casp3a- (live) cancer cell. Indeed, their "dead cell proximity index" (DCPI, i.e., the

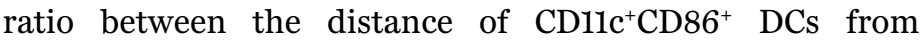
Casp3 $\mathrm{a}^{+}$cancer cells and the distance of $\mathrm{CD} 11 \mathrm{c}^{+} \mathrm{CD} 86^{+} \mathrm{DCs}$ from Casp3a $\mathrm{a}^{-}$cancer cells) was consistently $>1$. Conversely,

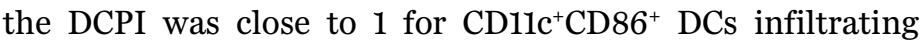
tumors treated with anthracyclines in $\mathrm{FprI}^{-1-}$ hosts (Fig. 3, $\mathrm{H}$ to $\mathrm{K}$ ). In accord with its expression pattern (Fig. 3B), FprI did not influence the DCPI of macrophages, yet affected that of neutrophils (fig. S17D). Thus, FPR1 determines the spatial positioning of DCs with respect to dying cancer cells. Accordingly, Fpr1 blockade with CsH or Fpr1 knockout reduced the capacity of intratumoral $\mathrm{CD} 11 \mathrm{~b}^{+} \mathrm{Ly} 6 \mathrm{C}^{\text {high }} \mathrm{Ly} 6 \mathrm{G}^{-}$ cells (which include inflammatory DCs) (28) to acquire antigens from cancers engineered to express green fluorescent protein (GFP) tethered to the inner leaflet of the plasma membrane, in response to chemotherapy (Fig. $4 \mathrm{~A}$ and fig. S18, A and B). Moreover, FprI inhibition interfered with the

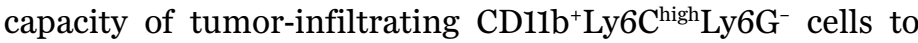
express increased levels of Class II MHC proteins (I-A/I-E) upon chemotherapy in vivo (Fig. 4B and fig. S18, A and B). However, Fpr1 knockout did not affect major DC functions in vitro and did not interfere with the presentation of soluble protein antigen by DC (figs. S19 and S20).

The failure of $\mathrm{FprI}^{-/-}$DCs to encounter dying cancer cells and to take up tumor antigens should compromise $\mathrm{T}$ cell responses. Accordingly, the capacity of chemotherapy to stimulate the intratumoral proliferation of adoptively transferred $\mathrm{T}$ cells specific for a model antigen (ovalbumin, OVA) was attenuated in $\mathrm{FprI}^{-/-}$mice harboring OVA-expressing MCA205 tumors, as compared to WT mice (fig. S21A). WT DCs purified from OVA-expressing tumors postchemotherapy stimulated OT1 responses upon their adoptive transfer into naïve mice, while $\mathrm{FprI}^{-/-}$DCs failed to do so (fig. S21, B and C). Moreover, the frequency of tumorinfiltrating $\mathrm{CD}^{+} \mathrm{T}$ cells was increased by anthracyclinebased chemotherapy in WT mice, but less so in $\mathrm{FprI}^{-1-}$ hosts (Fig. 4, C and D).

The aforementioned results suggest that the initial defect of the $\mathrm{FprI}^{-1-}$ immune system consists in the failure of DCs to migrate toward dying cancer cells. We attempted to explore this hypothesis in vitro. When mouse splenocytes or human peripheral blood mononuclear cells (PBMC) expressing functional FPRI (WT for mice, $F P R I^{C C}$ for humans) were confronted with anthracycline-treated WT cancer cells in a microfluidic device (29), they migrated toward dying/dead cells treated with doxorubicin (but not to live untreated cells), and the fraction of leukocytes containing DCs exhibited a prolonged ( $>60 \mathrm{~min}$ ) juxtaposition with the corpses (Fig. 4, E and F, and fig. S22A). Conversely, FPRI-deficient cells $\left(F p r 1^{-1-}\right.$ for mice, $F P R I^{C A}$ or $F P R I^{A A}$ for humans) were largely unable to engage in such long-term interactions. Moreover, no stable conjugates were formed between DCs and dying/dead $A n x a 1^{-1-}$ cancer cells (Fig. 4, E to J; fig. S22, A and B; fig. S23, A to C; and movies S1 to S24). Prolonged interactions of dying cancer cells with human blood-borne DCs were observed, but only if such DC expressed functional FPR1 (fig. S22, C and D).

Altogether, our findings underscore the obligate contribution of the interaction between ANXA1 and FPR1 to the immune response against cancer cells succumbing to chemotherapy. While it does not influence the recruitment of inflammatory DCs (with a CD11c ${ }^{+} \mathrm{CD} 86^{+} \mathrm{Ly} 6 \mathrm{C}^{\text {high }}$ phenotype) to the tumor bed (which depends on other factors, including ATP and chemokines) $(26,28)$, FPR1 is required for DCs to come into close proximity of dying cancer cells, to establish stable contacts with corpses, to take up tumor-associated antigens, and to cross-present them to T cells. Hence, deficient FPR1 signaling results in defective intratumoral DC maturation and insufficient $\mathrm{T}$ cell-mediated anticancer immune responses, in fine abolishing the efficacy of chemotherapy. Given the high allelic frequency of the loss-offunction FPR1 SNP rs867228 (17), it will be important to identify means for restoring or bypassing defective FPR1 signaling.

\section{REFERENCES AND NOTES}

1. L. Zitvogel, L. Galluzzi, M. J. Smyth, G. Kroemer, Mechanism of action of conventional and targeted anticancer therapies: Reinstating immunosurveillance. Immunity 39, 74-88 (2013).__ Medline doi:10.1016/j.immuni.2013.06.014

2. A. Sklan, Lymphocytes prognostic in breast cancer. Lancet Oncol. 15, e422 (2014). Medline doi:10.1016/S1470-2045(14)70369-9

3. C. Denkert, S. Loibl, A. Noske, M. Roller, B. M. Müller, M. Komor, J. Budczies, S. Darb-Esfahani, R. Kronenwett, C. Hanusch, C. von Törne, W. Weichert, K. Engels, C. Solbach, I. Schrader, M. Dietel, G. von Minckwitz, Tumor-associated lymphocytes as an independent predictor of response to neoadjuvant chemotherapy in breast cancer. J. Clin. Oncol. 28, 105-113 (2010). Medline doi:10.1200/JC0.2009.23.7370

4. M. L. Broz, M. Binnewies, B. Boldajipour, A. E. Nelson, J. L. Pollack, D. J. Erle, A. Barczak, M. D. Rosenblum, A. Daud, D. L. Barber, S. Amigorena, L. J. Van't Veer, A. I. Sperling, D. M. Wolf, M. F. Krummel, Dissecting the tumor myeloid compartment reveals rare activating antigen-presenting cells critical for $T$ cell immunity. Cancer Cell 26, 638-652 (2014).__ Medline doi:10.1016/j.ccell.2014.09.007

5. L. Senovilla, E. Vacchelli, J. Galon, S. Adjemian, A. Eggermont, W. H. Fridman, C. Sautès-Fridman, Y. Ma, E. Tartour, L. Zitvogel, G. Kroemer, L. Galluzzi, Trial watch: Prognostic and predictive value of the immune infiltrate in cancer. Oncolmmunology 1, 1323-1343 (2012). Medline doi:10.4161/onci.22009

6. L. Senovilla, I. Vitale, I. Martins, M. Tailler, C. Pailleret, M. Michaud, L. Galluzzi, S. Adjemian, O. Kepp, M. Niso-Santano, S. Shen, G. Mariño, A. Criollo, A. Boilève, B. Job, S. Ladoire, F. Ghiringhelli, A. Sistigu, T. Yamazaki, S. Rello-Varona, C. Locher, V. Poirier-Colame, M. Talbot, A. Valent, F. Berardinelli, A. Antoccia, F. 
Ciccosanti, G. M. Fimia, M. Piacentini, A. Fueyo, N. L. Messina, M. Li, C. J. Chan, V. Sigl, G. Pourcher, C. Ruckenstuhl, D. Carmona-Gutierrez, V. Lazar, J. M. Penninger, F. Madeo, C. López-Otín, M. J. Smyth, L. Zitvogel, M. Castedo, G. Kroemer, An immunosurveillance mechanism controls cancer cell ploidy. Science 337, 1678-1684 (2012). Medline

7. L. Apetoh, F. Ghiringhelli, A. Tesniere, M. Obeid, C. Ortiz, A. Criollo, G. Mignot, M. C. Maiuri, E. Ullrich, P. Saulnier, H. Yang, S. Amigorena, B. Ryffel, F. J. Barrat, P. Saftig, F. Levi, R. Lidereau, C. Nogues, J. P. Mira, A. Chompret, V. Joulin, F. Clavel-Chapelon, J. Bourhis, F. André, S. Delaloge, T. Tursz, G. Kroemer, L. Zitvogel, Toll-like receptor 4-dependent contribution of the immune system to anticancer chemotherapy and radiotherapy. Nat. Med. 13, 1050-1059 (2007). Medline doi:10.1038/nm1622

8. G. Kroemer, L. Senovilla, L. Galluzzi, F. André, L. Zitvogel, Natural and therapyinduced immunosurveillance in breast cancer. Nat. Med. 21, 1128-1138 (2015). Medline doi:10.1038/nm.3944

9. A. Sistigu, T. Yamazaki, E. Vacchelli, K. Chaba, D. P. Enot, J. Adam, I. Vitale, A. Goubar, E. E. Baracco, C. Remédios, L. Fend, D. Hannani, L. Aymeric, Y. Ma, M. Niso-Santano, O. Kepp, J. L. Schultze, T. Tüting, F. Belardelli, L. Bracci, V. La Sorsa, G. Ziccheddu, P. Sestili, F. Urbani, M. Delorenzi, M. Lacroix-Triki, V. Quidville, R. Conforti, J. P. Spano, L. Pusztai, V. Poirier-Colame, S. Delaloge, F. Penault-Llorca, S. Ladoire, L. Arnould, J. Cyrta, M. C. Dessoliers, A. Eggermont, M. E. Bianchi, M. Pittet, C. Engblom, C. Pfirschke, X. Préville, G. Uzè, R. D. Schreiber, M. T. Chow, M. J. Smyth, E. Proietti, F. André, G. Kroemer, L. Zitvogel, Cancer cell-autonomous contribution of type I interferon signaling to the efficacy of chemotherapy. Nat. Med. 20, 1301-1309 (2014)._Medline doi:10.1038/nm.3708

10. G. Kroemer, L. Galluzzi, O. Kepp, L. Zitvogel, Immunogenic cell death in cancer therapy. Annu. Rev. Immunol. 31, 51-72 (2013). Medline doi:10.1146/annurevimmunol-032712-100008

11. L. Zitvogel, O. Kepp, G. Kroemer, Decoding cell death signals in inflammation and immunity. Cell 140, 798-804 (2010). Medline doi:10.1016/j.cell.2010.02.015

12. P. E. Marques, S. S. Amaral, D. A. Pires, L. L. Nogueira, F. M. Soriani, B. H. Lima, G. A. Lopes, R. C. Russo, T. V. Avila, J. G. Melgaço, A. G. Oliveira, M. A. Pinto, C. X. Lima, A. M. De Paula, D. C. Cara, M. F. Leite, M. M. Teixeira, G. B. Menezes, Chemokines and mitochondrial products activate neutrophils to amplify organ injury during mouse acute liver failure. Hepatology 56, 1971-1982 (2012). Medline

13. B. McDonald, K. Pittman, G. B. Menezes, S. A. Hirota, I. Slaba, C. C. Waterhouse, P. L. Beck, D. A. Muruve, P. Kubes, Intravascular danger signals guide neutrophils to sites of sterile inflammation. Science 330, 362-366 (2010). Medline doi:10.1126/science.1195491

14. R. Seifert, K. Wenzel-Seifert, The human formyl peptide receptor as model system for constitutively active G-protein-coupled receptors. Life Sci. 73, 22632280 (2003). Medline doi:10.1016/S0024-3205(03)00654-4

15. K. Wenzel-Seifert, R. Seifert, Functional differences between human formyl peptide receptor isoforms 26, 98, and G6. Naunyn Schmiedebergs Arch. Pharmacol. 367, 509-515 (2003). Medline doi:10.1007/s00210-003-0714-7

16. H. M. Miettinen, Regulation of human formyl peptide receptor 1 synthesis: Role of single nucleotide polymorphisms, transcription factors, and inflammatory mediators. PLOS ONE 6, e28712 (2011)._Medline doi:10.1371/journal.pone.0028712

17. A. Sahagun-Ruiz, J. S. Colla, J. Juhn, J. L. Gao, P. M. Murphy, D. H. McDermott, Contrasting evolution of the human leukocyte $\mathrm{N}$-formylpeptide receptor subtypes FPR and FPRL1R. Genes Immun. 2, 335-342 (2001). Medline doi:10.1038/sj.gene. 6363787

18. F. Ghiringhelli, L. Apetoh, A. Tesniere, L. Aymeric, Y. Ma, C. Ortiz, K. Vermaelen, T. Panaretakis, G. Mignot, E. Ullrich, J. L. Perfettini, F. Schlemmer, E. Tasdemir, M. Uhl, P. Génin, A. Civas, B. Ryffel, J. Kanellopoulos, J. Tschopp, F. André, R. Lidereau, N. M. McLaughlin, N. M. Haynes, M. J. Smyth, G. Kroemer, L. Zitvogel, Activation of the NLRP3 inflammasome in dendritic cells induces IL-1betadependent adaptive immunity against tumors. Nat. Med. 15, 1170-1178 (2009). Medline doi:10.1038/nm.2028

19. N. Prevete, F. Liotti, C. Visciano, G. Marone, R. M. Melillo, A. de Paulis, The formyl peptide receptor 1 exerts a tumor suppressor function in human gastric cancer by inhibiting angiogenesis. Oncogene 34, 3826-3838 (2015). Medline doi:10.1038/onc.2014.309

20. R. Sun, P. Iribarren, N. Zhang, Y. Zhou, W. Gong, E. H. Cho, S. Lockett, O. Chertov, F. Bednar, T. J. Rogers, J. J. Oppenheim, J. M. Wang, Identification of neutrophil granule protein cathepsin $G$ as a novel chemotactic agonist for the $G$ proteincoupled formyl peptide receptor. J. Immunol. 173, 428-436 (2004). Medline doi:10.4049/jimmunol.173.1.428

21. W. Wang, T. Li, X. Wang, W. Yuan, Y. Cheng, H. Zhang, E. Xu, Y. Zhang, S. Shi, D. Ma, W. Han, FAM19A4 is a novel cytokine ligand of formyl peptide receptor 1 (FPR1) and is able to promote the migration and phagocytosis of macrophages. Cell. Mol. Immunol. 12, 615-624 (2015). Medline doi:10.1038/cmi.2014.61

22. E. J. Tucker, S. G. Hershman, C. Köhrer, C. A. Belcher-Timme, J. Patel, O. A. Goldberger, J. Christodoulou, J. M. Silberstein, M. McKenzie, M. T. Ryan, A. G. Compton, J. D. Jaffe, S. A. Carr, S. E. Calvo, U. L. RajBhandary, D. R. Thorburn, V. K. Mootha, Mutations in MTFMT underlie a human disorder of formylation causing impaired mitochondrial translation. Cell Metab. 14, 428-434 (2011). Medline doi:10.1016/j.cmet.2011.07.010

23. Y. Li, D. Ye, Molecular biology for formyl peptide receptors in human diseases. J. Mol. Med. 91, 781-789 (2013). Medline doi:10.1007/s00109-013-1005-5

24. J. Huang, K. Chen, J. Chen, W. Gong, N. M. Dunlop, O. M. Howard, Y. Gao, X. W. Bian, J. M. Wang, The G-protein-coupled formylpeptide receptor FPR confers a more invasive phenotype on human glioblastoma cells. Br. J. Cancer 102, 10521060 (2010). Medline doi:10.1038/sj.bjc.6605591

25. A. L. Stenfeldt, J. Karlsson, C. Wennerås, J. Bylund, H. Fu, C. Dahlgren, Cyclosporin $\mathrm{H}$, Boc-MLF and Boc-FLFLF are antagonists that preferentially inhibit activity triggered through the formyl peptide receptor. Inflammation 30 , 224-229 (2007). Medline doi:10.1007/s10753-007-9040-4

26. Y. Ma, S. Adjemian, S. R. Mattarollo, T. Yamazaki, L. Aymeric, H. Yang, J. P. Portela Catani, D. Hannani, H. Duret, K. Steegh, I. Martins, F. Schlemmer, M. Michaud, O. Kepp, A. Q. Sukkurwala, L. Menger, E. Vacchelli, N. Droin, L. Galluzzi, R. Krzysiek, S. Gordon, P. R. Taylor, P. Van Endert, E. Solary, M. J. Smyth, L. Zitvogel, G. Kroemer, Anticancer chemotherapy-induced intratumoral recruitment and differentiation of antigen-presenting cells. Immunity 38, 729741 (2013). Medline doi:10.1016/j.immuni.2013.03.003

27. A. Boissonnas, L. Fetler, I. S. Zeelenberg, S. Hugues, S. Amigorena, In vivo imaging of cytotoxic $T$ cell infiltration and elimination of a solid tumor. J. Exp. Med. 204, 345-356 (2007). Medline doi:10.1084/jem.20061890

28. Y. Ma, S. R. Mattarollo, S. Adjemian, H. Yang, L. Aymeric, D. Hannani, J. P. Portela Catani, H. Duret, M. W. Teng, O. Kepp, Y. Wang, A. Sistigu, J. L. Schultze, G. Stoll, L. Galluzzi, L. Zitvogel, M. J. Smyth, G. Kroemer, CCL2/CCR2-dependent recruitment of functional antigen-presenting cells into tumors upon chemotherapy. Cancer Res. 74, 436-445 (2014). Medline

29. L. Businaro, A. De Ninno, G. Schiavoni, V. Lucarini, G. Ciasca, A. Gerardino, F. Belardelli, L. Gabriele, F. Mattei, Cross talk between cancer and immune cells: Exploring complex dynamics in a microfluidic environment. Lab Chip 13, 229239 (2013). Medline doi:10.1039/C2LC40887B

30. Y. Hochberg, Y. Benjamini, More powerful procedures for multiple significance testing. Stat. Med. 9, 811-818 (1990). Medline doi:10.1002/sim.4780090710

31. M. Slatkin, Linkage disequilibrium: Understanding the evolutionary past and mapping the medical future. Nat. Rev. Genet. 9, 477-485 (2008)._Medline doi:10.1038/nrg2361

32. V. Boige, J. Mendiboure, J. P. Pignon, M. A. Loriot, M. Castaing, M. Barrois, D. Malka, D. A. Trégouët, O. Bouché, D. Le Corre, I. Miran, C. Mulot, M. Ducreux, P. Beaune, P. Laurent-Puig, Pharmacogenetic assessment of toxicity and outcome in patients with metastatic colorectal cancer treated with LV5FU2, FOLFOX, and FOLFIRI: FFCD 2000-05. J. Clin. Oncol. 28, 2556-2564 (2010)._Medline doi:10.1200/JC0.2009.25.2106

33. E. Sugar, A. J. Pascoe, N. Azad, Reporting of preclinical tumor-graft cancer therapeutic studies. Cancer Biol. Ther. 13, 1262-1268 (2012)._Medline doi:10.4161/cbt.21782

34. E. Demidenko, The assessment of tumour response to treatment. Journal of the Royal Statistical Society: Series C 55, 365-377 (2006). doi:10.1111/j.14679876.2006.00541.x

35. J. Schindelin, I. Arganda-Carreras, E. Frise, V. Kaynig, M. Longair, T. Pietzsch, S. Preibisch, C. Rueden, S. Saalfeld, B. Schmid, J. Y. Tinevez, D. J. White, V. Hartenstein, K. Eliceiri, P. Tomancak, A. Cardona, Fiji: An open-source platform for biological-image analysis. Nat. Methods 9, 676-682 (2012)._Medline doi:10.1038/nmeth.2019

36. S. Maere, K. Heymans, M. Kuiper, BiNGO: A Cytoscape plugin to assess overrepresentation of gene ontology categories in biological networks. Bioinformatics 21, 3448-3449 (2005)._Medline doi:10.1093/bioinformatics/bti551 
37. B. Efron, R. Tibshirani, On testing the significance of sets of genes. Ann. Appl. Stat. 1, 107-129 (2007). doi:10.1214/07-AOAS101

38. Cancer Genome Atlas Network, Comprehensive molecular portraits of human breast tumours. Nature 490, 61-70 (2012). Medline doi:10.1038/nature11412

39. A. Subramanian, P. Tamayo, V. K. Mootha, S. Mukherjee, B. L. Ebert, M. A. Gillette, A. Paulovich, S. L. Pomeroy, T. R. Golub, E. S. Lander, J. P. Mesirov, Gene set enrichment analysis: A knowledge-based approach for interpreting genomewide expression profiles. Proc. Natl. Acad. Sci. U.S.A. 102, 15545-15550 (2005). Medline doi:10.1073/pnas.0506580102

40. C. A. Schneider, W. S. Rasband, K. W. Eliceiri, NIH Image to Image J: 25 years of image analysis. Nat. Methods 9, 671-675 (2012)._Medline doi:10.1038/nmeth.2089

\section{ACKNOWLEDGMENTS}

The data presented in this manuscript are tabulated in the main paper and in the supplementary materials. L.Z. filed a patent (W02011131472 A1) that relates to the predictive role of single-nucleotide polymorphisms in cancer therapy. G.K. and L.Z. are supported by the Ligue Nationale contre le Cancer (Equipes labelisées), Sites de Recherche Intégrées sur le Cancer (SIRIC) Socrates and Carpem, the ISREC Foundation, Agence Nationale pour la Recherche (AUTOPH, Emergence), Cancéropôle lle-de-France, European Commission (ArtForce), European Research Council Advanced Investigator Grant (to G.K.), Fondation pour la Recherche Médicale, Fondation de France, the LabEx Immuno-Oncology, Institut National du Cancer (INCa), and the Paris Alliance of Cancer Research Institutes. Y.M. is supported by the LabEx Immuno-Oncologie and the Chinese National Thousand Talents Program; H.Y. by the Chinese National Thousand Talents Program; J.L.S. by the Deutsche Forschungsgemeinschaft (SFB704, SFB645) and the Excellence Cluster ImmunoSensation; F.M. by Italian Ministry of Health (RF-2011-02347120); G.S. by Associazione italiana per la ricerca sul cancro (AIRC) (IG 14297). I.V. by AIRC (MFAG 14641), Italian Ministry of Health (RF_GR 2011-2012) and Programma per i Giovani Ricercatori "Rita Levi Montalcini" 2011; A.S. and G.M. by AIRC.

\section{SUPPLEMENTARY MATERIALS}

www.sciencemag.org/cgi/content/full/science.aad0779/DC1

Materials and Methods

Figs. S1 to S23

Tables S1 to S11

Movies S1 to S24

References (30-40)

26 July 2015; accepted 8 October 2015

Published online 29 October 2015

10.1126/science.aad0779 

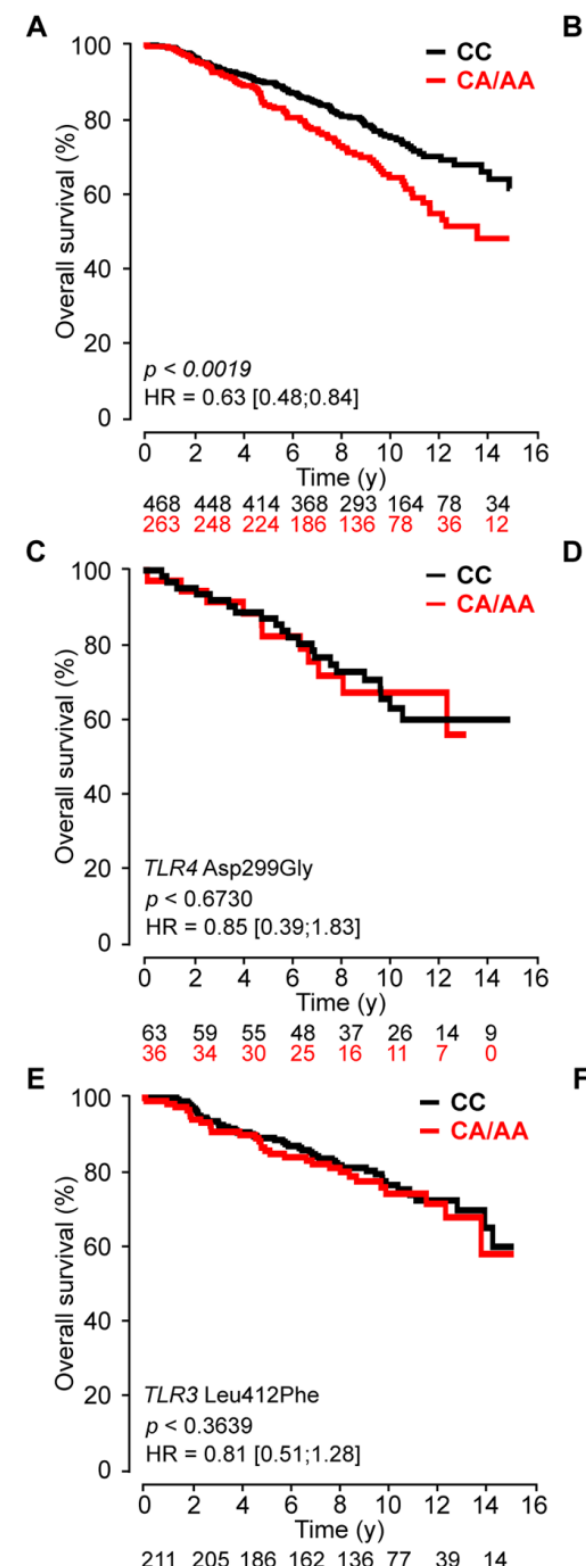

$\begin{array}{llllllll}211 & 205 & 186 & 162 & 136 & 77 & 39 & 14 \\ 123 & 116 & 108 & 94 & 76 & 43 & 24 & 5\end{array}$
B

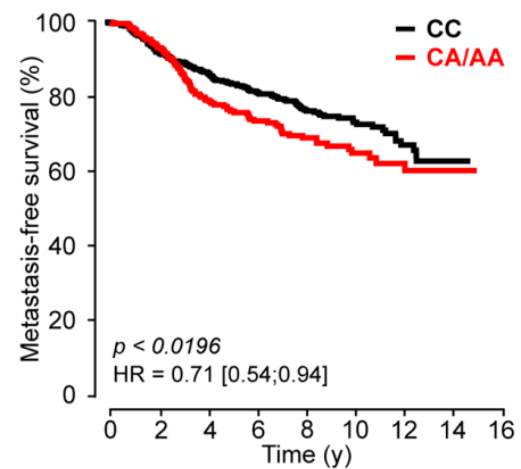

$\begin{array}{llllllll}468 & 428 & 384 & 334 & 263 & 147 & 66 & 29 \\ 263 & 236 & 192 & 158 & 112 & 65 & 32 & 12\end{array}$

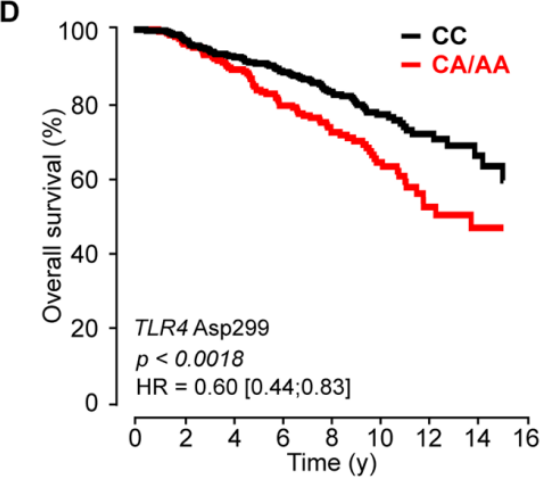

$38036733830223912658 \quad 25$

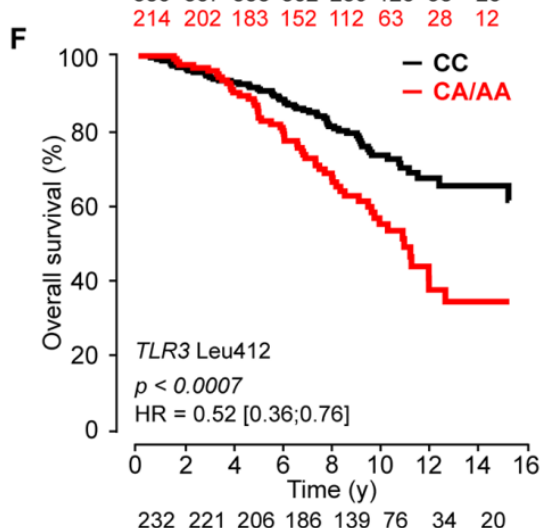

$\begin{array}{llllllll}232 & 221 & 206 & 186 & 139 & 76 & 34 & 20\end{array}$

Fig. 1. Effects of FPR1 deficiency on the survival of breast cancer patients. (A and B) Kaplan-Meier of the overall survival (OS) (A) or metastasis-free survival (MFS) (B) estimated in the combined cohort of patients $(n=731)$ treated with adjuvant anthracycline-based chemotherapy and bearing FPR1 rs 867228 with CC (wild type) or CA (heterozygous) + AA (mutated homozygous) genotypes. The analysis of the effect of FPR1 rs867228 on OS yields a false discovery rate of 0.1090. (C and D) OS estimated in breast cancer patients bearing mutated (Asp299Gly) (C) or wild type (Asp299Asp) (D) Toll like receptor 4 (TLR4) (SNP rs4986790) genotypes according to the illustrated FPR1 SNP rs867228 genotype. (E and F) Kaplan-Meier of the OS estimated in breast cancer patients bearing mutated (Leu412Phe) (E) or wild type (Leu412Leu) (F) TLR3 genotypes (SNP rs3775291) according to the FPR1 SNP rs867228 genotype. Statistical significance was determined by likelihood ratio test (LRT). HR, hazard ratio. 
A

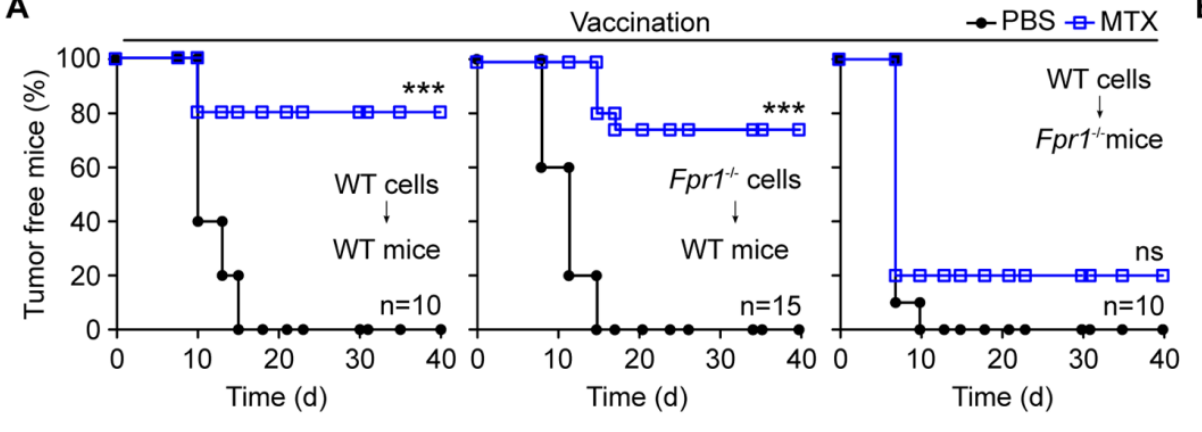

C

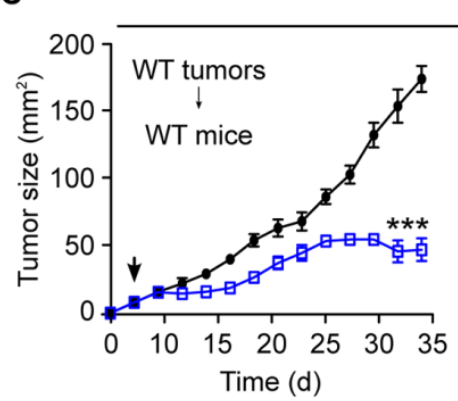

E

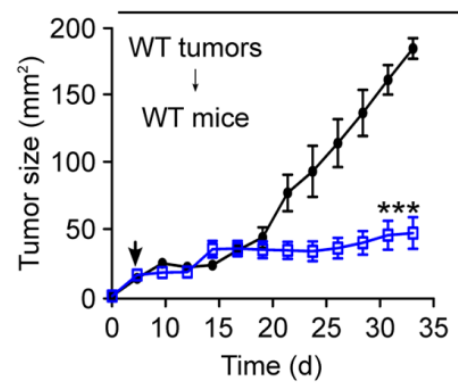

Chemotherapy

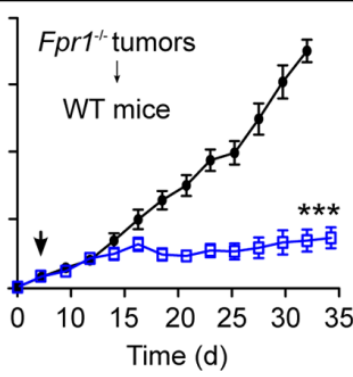

Chemotherapy

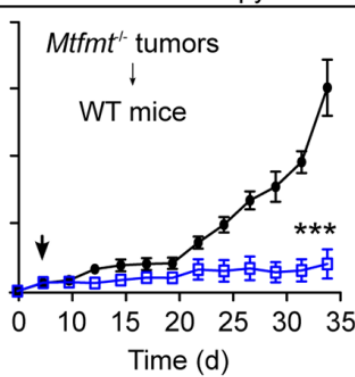

Time (d) $\rightarrow$ PBS $\square$ MTX

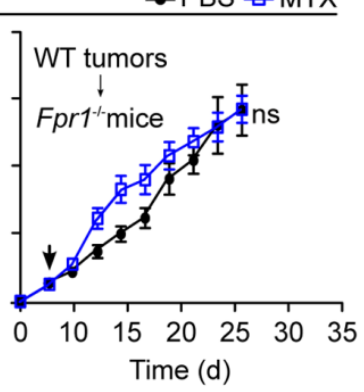

$\rightarrow$ PBS $¥ \mathrm{MTX}$

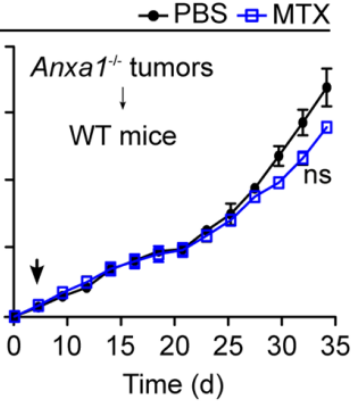

B

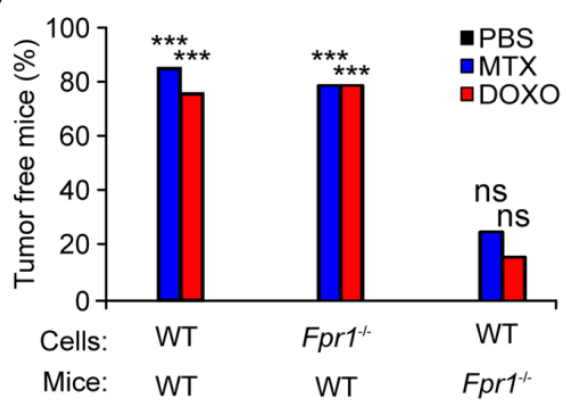

D

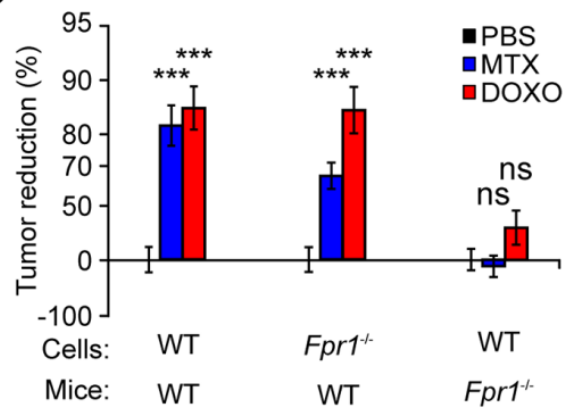

$\mathbf{F}$

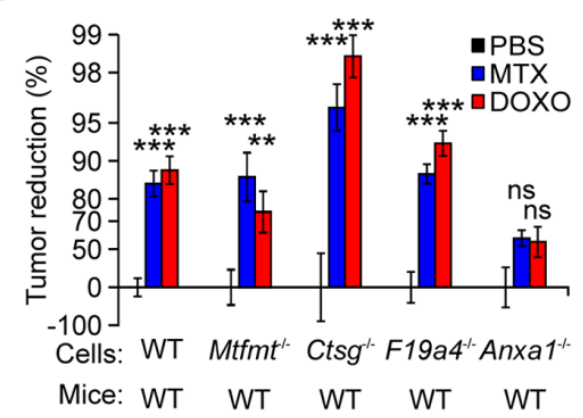

Fig. 2. Contribution of Fprl and Annexin A1 to chemotherapy-induced tumor growth reduction. (A and B) WT or Fpr1 ${ }^{-1-}$ MCA205 fibrosarcoma cells cultured overnight with mitoxantrone (MTX) or doxorubicin (DOXO) were inoculated s.c. into the flanks of WT C57BL/6 or Fpr1 ${ }^{-1-}$ mice. Seven days later animals were rechallenged into the opposite flank with live WT MCA205 cells. Representative experiment (A) and quantitative analysis of the data [in (A) and fig. S5A) in (B)]. (C and D) WT and Fpr1 ${ }^{-/-}$C57BL/6 mice bearing WT or Fpr1 ${ }^{-/-}$MCA205 fibrosarcomas were treated with MTX, DOXO or PBS (as a vehicle control). Representative experiment in (C) and quantitative analysis of the data [in (C) and fig. S5B]. (E and F) WT, Mtfmt ${ }^{-/-}, \mathrm{Ctsg}^{-/-}, \mathrm{FlOa}^{-/ 4^{--}}$or Anxa1 ${ }^{-/-}$ MCA205 tumors were established in WT mice and treated with MTX, DOXO or PBS (as a vehicle control). Results are representative for one experiment out of three involving at least five mice per group (A, C, E) or are pooled from at least three independent experiments (B, D, F). Statistical significance was calculated by means of the likelihood ratio test $(A)$, the Chi square test $(B)$, the Wald test $(C, E)$ and the contrast $t$ test $(D, F)$ when comparing to PBS controls. ns, not significant, ${ }^{* *} P<0.001$. Arrows in $(C)$ and $(E)$ indicate the initiation of chemotherapy. 
A

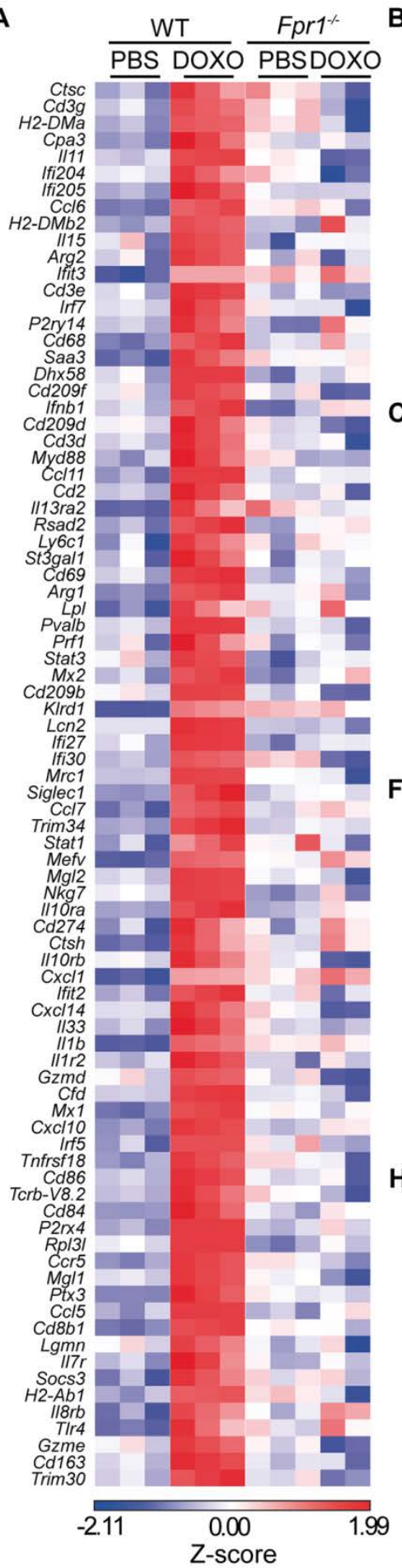

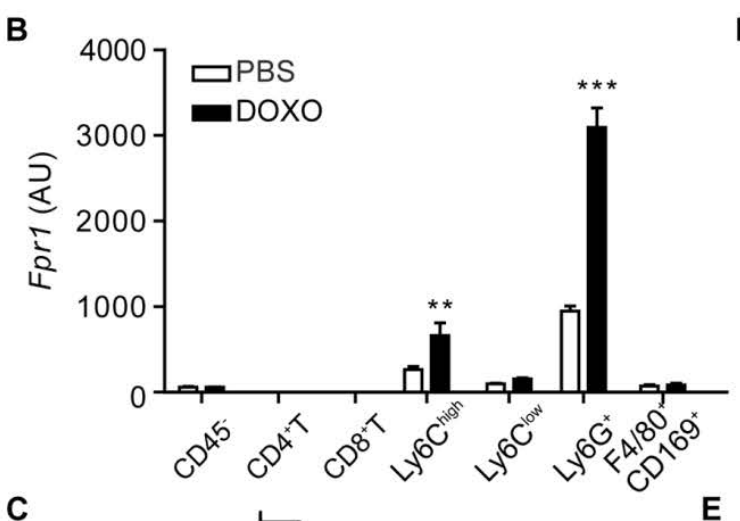
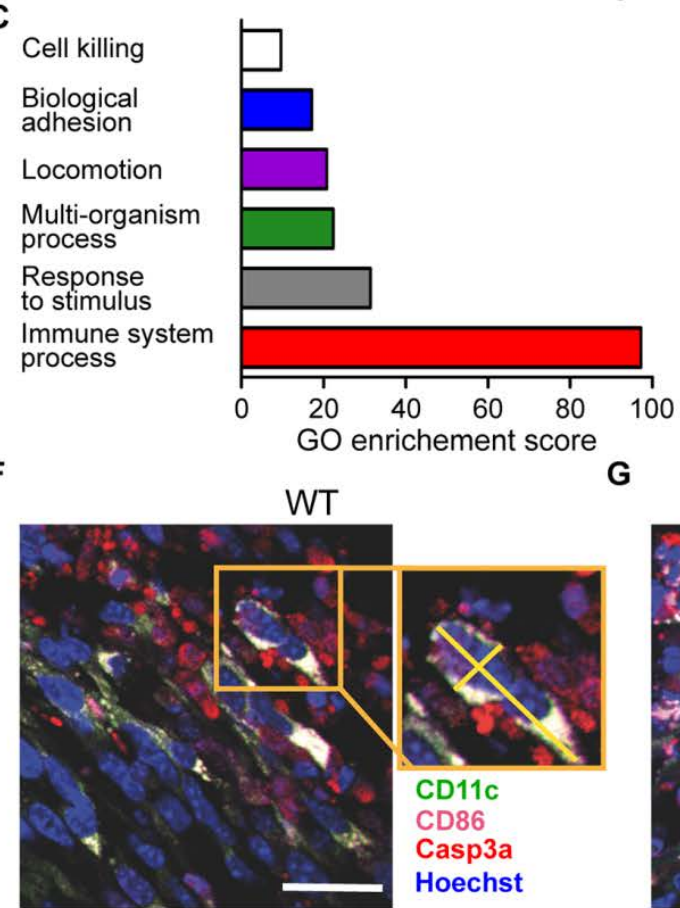

H

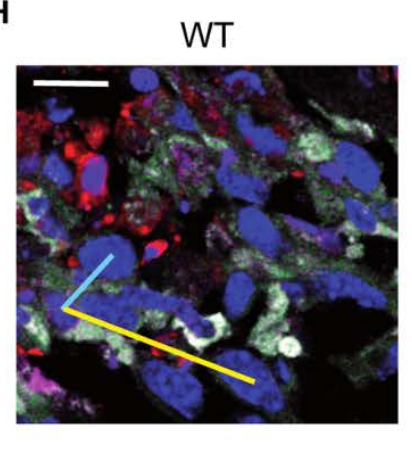

D
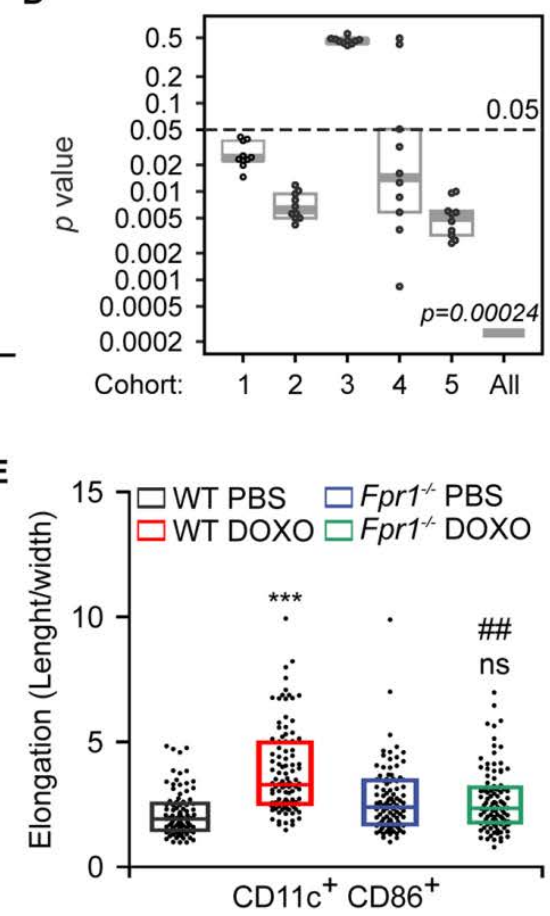

G

$\mathrm{Fpr}^{-1}$

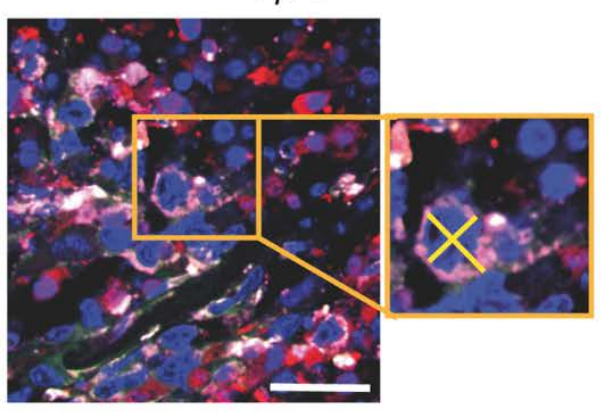

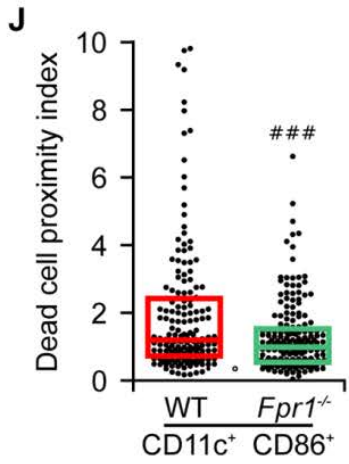


Fig. 3. Impact of FPR1 on the anticancer immune response and the intratumoral positioning of dendritic cells post-chemotherapy. (A) WT or Fpr1 ${ }^{-1-}$ mice bearing murine MCA205 fibrosarcomas were treated with doxorubicin (DOXO) or PBS, and tumors were harvested 48 hours later for microarray analysis. Immunerelated genes upregulated by DOXO administration in tumors from WT mice (but not from Fpr1 ${ }^{-/-}$mice) are listed. (B) Alternatively, tumors were recovered and tumor infiltrating immune cells were sorted by FACS. Fpr1 expression was assessed by quantitative real time (RT)-PCR. AU, arbitrary unit. ns, non significant, ${ }^{* *} P<$ 0.01 , ${ }^{* *} P<0.001$ (unpaired $t$ test) as compared to PBS administration. (C) A total of 427 genes, identified as highly expressed specifically in DOXO-treated MCA205 tumors growing in WT mice (but not in $\mathrm{Fpr}^{-{ }^{--}}$mice), were subjected to gene ontology analysis. (D) Analysis of the overexpression of the set of 84 immune-related genes identified in (A) on "responsive" as compared to "non-responsive" tumors from 5 independent breast cancer patient cohorts, by employing the Gene Set Analysis (GSA). The combined (All) $P$ value was obtained using Fisher's method. The vertical dotted line represents significance thresholds $(P<0.05)$. (E to $G)$ Impact of chemotherapy with DOXO on the immune infiltrate of MCA205 fibrosarcomas. Immunofluorescence stainings were performed on tumors recovered two days post-chemotherapy Quantitative analysis (E) of the immunostainings ( $F, G$ ) are shown. In (E) the length/width ratio (elongation) was determined in 60-100 randomly chosen cells, using the morphometric methods illustrated in ( $F, G)$. Boxplots report the lower and upper quartile plus the median value. ns, non significant, ${ }^{* *} P<0.001$, one way ANOVA, Bonferroni's Multiple Comparison Test as compared to tumors harvested from mice with the same genetic background and receiving PBS; \#\#P < 0.01, as compared to tumors harvested from WT mice treated with DOXO. (F, G) Representative fluorescence micrographs of tumor-infiltrating dendritic cells (DCs, CD11c $\mathrm{C}^{+} \mathrm{CD6}{ }^{+}$) in DOXOtreated, dead cell $\left(\mathrm{Casp} 3 \mathrm{a}^{+}\right)$enriched tumor sites are reported. Enlarged windows describe the method of length/width quantification. Scale bar $10 \mu \mathrm{m}$. ( $\mathrm{H}$ and $\mathrm{I})$ Representative images showing the method employed to measure the intercellular distance between the same DC $\left(C D 11 c^{+} C D 86^{+}\right)$and the closest Casp3a- (blue line) or Casp3a+ (yellow line) DOXO-treated tumor cells. Scale bar $20 \mu \mathrm{m}$. (J) Quantitative analysis of the dead cell proximity index determined for tumor-infiltrating DCs. Data are represented as scatter dot plots. $\# \# \# P<0.001$ ( $t$ test) as compared to tumor cells from MTX-treated WT mice. 
A

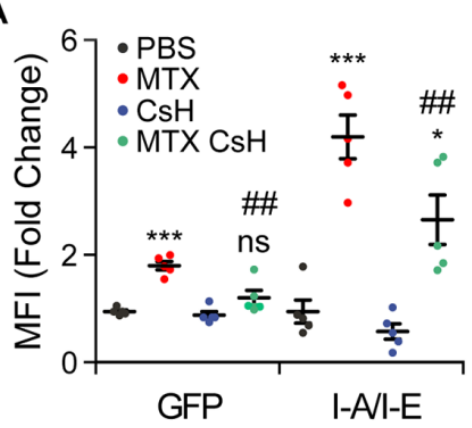

E

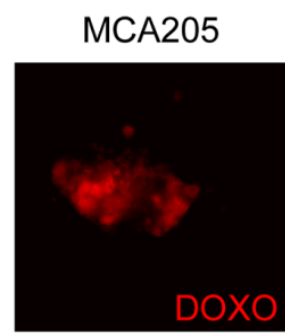

G
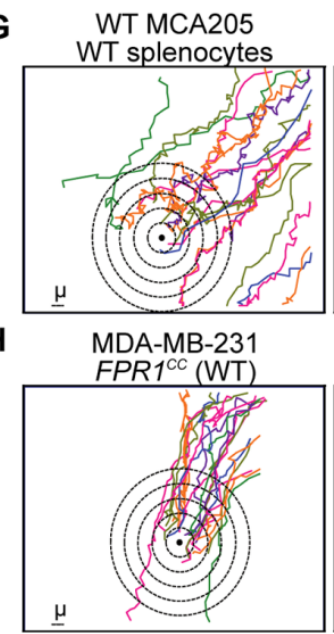

B

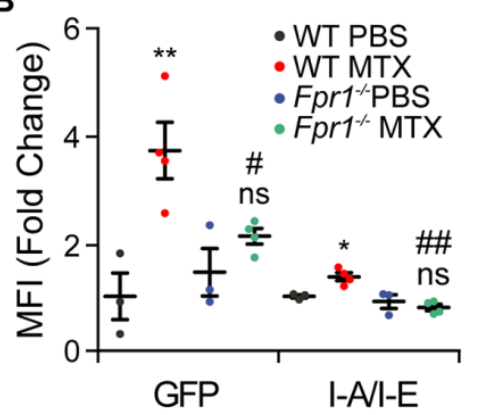

C

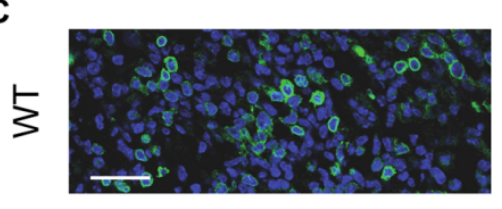

$\frac{1}{2}$

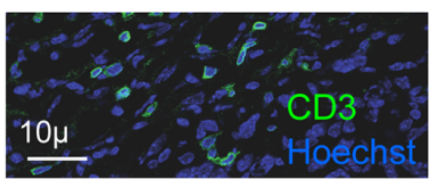

$\mathbf{F}$

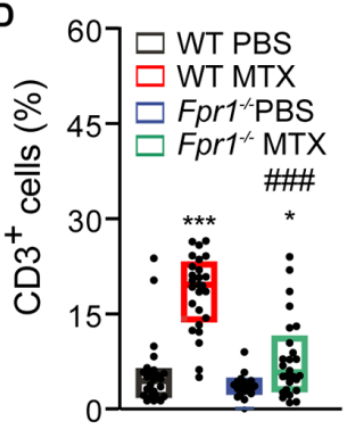

MDA-MB-231
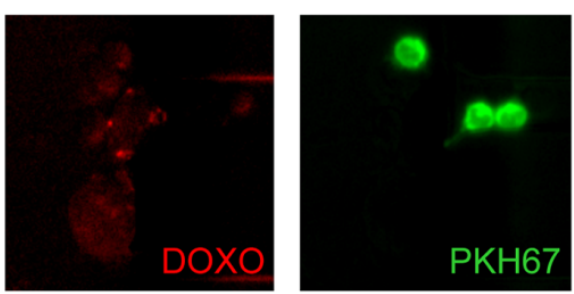

$\mathbf{J}$

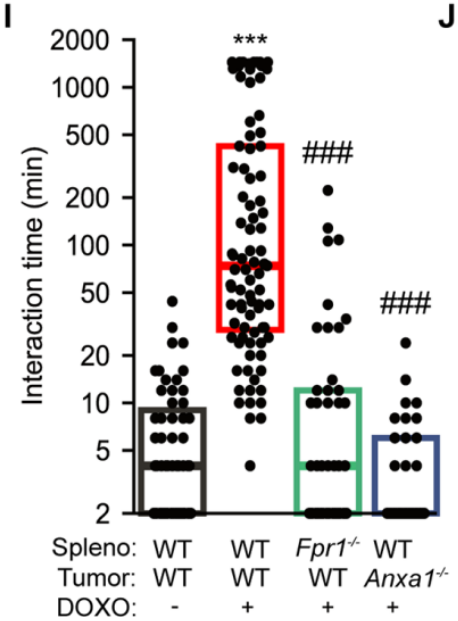

Merged
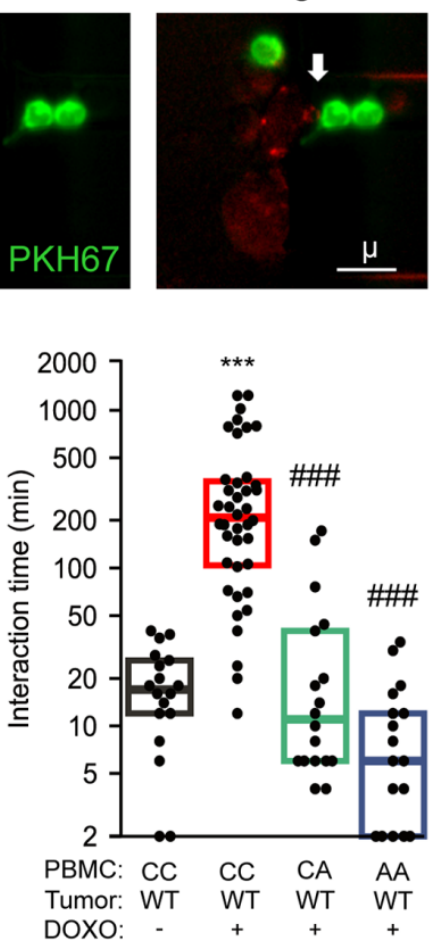
Fig. 4. Impact of FPR1 on dendritic cell function. (A and B) MyrPalm-green fluorescent protein (GFP)expressing MCA205 fibrosarcoma tumors were implanted in WT (A) or Fpr1 ${ }^{-/-}$mice $(B)$ and treated with mitoxantrone (MTX) or PBS as a vehicle control, alone or in combination with cyclosporin $\mathrm{H}(\mathrm{CsH})(\mathrm{A})$. Thirty-eight hours later, tumor infiltrating $C D 11 b^{+} L y 6 C^{\text {high }} L y 6 G^{-}$myeloid cells were isolated and assessed for tumor antigen uptake (indicated by GFP fluorescence intensity) or MHC class II (I-A/I-E) expression. Representative mean fluorescence intensities (MFI) from 3 independent experiments are shown. ns, non significant, ${ }^{*} P<0.05,{ }^{* *} P<0.01,{ }^{* * *} P<0.001$ (One-way ANOVA Bonferroni's Multiple Comparison Test) as compared to tumors recovered from WT mice subjected to the same treatment but not exposed to MTX (A) or from mice with the same genetic background receiving PBS (B); \#P<0.05, \#\#P<0.01 as compared to tumors harvested from WT mice treated with MTX alone. (C and D) Immunofluorescence detection of $\mathrm{CD}^{+}$T cells in MCA205 tumors harvested from WT and $\mathrm{Fpr}^{-1-}$ mice 7 days after doxorubicin (DOXO) treatment. Representative fluorescence micrographs are shown in (C), and quantitative data are reported in (D). ${ }^{*} P<0.05,{ }^{* *} P<0.001$ (One way ANOVA, Bonferroni's Multiple Comparison Test) as compared to tumors harvested from mice with the same genetic background receiving PBS; \#\#\#P < 0.001 as compared to tumors harvested from WT mice treated with DOXO. (E and F) Representative fluorescence micrographs showing the interaction between MCA205-DOXO corpses (in red) and mouse dendritic cell (DC, purified by means of CD11c microbeads and labeled with the PKH67 green fluorescent cell tracker) (E) or MDA-MB-231-DOXO corpses (in red) and human DC (peripheral blood mononucleated cell, PBMC, labeled with PKH67) (F). White arrows indicate portions of DOXO corpses interacting with the DC. ( $G$ and $\mathrm{H}$ ) Microfluidic time-lapse recordings of the interaction between DOXO-pretreated WT or Anxa1 $1^{-/-}$MCA205 cells and WT or Fpr1 ${ }^{-/-}$splenocytes (G) or, alternatively, between DOXO-pretreated human MDA-MB-231 breast cancer cells and peripheral blood mononuclear cell (PBMC) collected from healthy donors bearing FPR1 CC (WT), CA (heterozygous, HT) or AA (mutated homozygous, MT) genotypes $(\mathrm{H})$. Plots represent the trajectories of the individual splenocytes or PBMC $(n=6-30$ cells per condition) toward target tumor cells (black spots) within 24-48h (splenocytes) or 48-72h (PBMC). (I and J) Quantification of the interaction times between WT or Fpr1 ${ }^{-/-}$splenocytes and WT or Anxa1 ${ }^{-/-}$MCA205 cells $(\mathrm{I})$ or between FPR1 ${ }^{C C}, F P R 1^{C A}$ or FPR1 ${ }^{A A}$ PBMC and MDA-MB-231 cells $(\mathrm{J})$ that had been exposed or not to DOXO. Each dot represents a single spleen cell $(n=38-97$ cells per condition) or a single PBMC $(n=$ 20-40 cells per condition). ${ }^{* *} P<0.001$ (Mann Whitney test) referred to WT splenocytes (I) or FPR1 ${ }^{C C}$ PBMC $(J)$ challenged with untreated tumor cells; \#\#\#P<0.001 compared to WT splenocytes (I) or FPR1 ${ }^{C C}$ PBMC $(J)$ challenged with DOXO-treated WT tumor cells. 